Erratum

\title{
Optimal foraging of a herivorous lizard, the green iguana in a seasonal environment
}

\section{Wouter D. van Marken Lichenbelt}

Carmabi Foundation, P.O. Box 2090, Curaçao, Netherlands Antilles; Zoological Laboratory, University of Groningen, PO Box 14, NL-9750 NN Haren, The Netherlands

Oecologia (1993) 95:246-256

Due to a technical error, figures 4 and 5 appeared in the wrong order. We apologize for any inconvenience caused. 